Nullius in verba: "Canakinumab for the Treatment of Autoinflammatory Recurrent Fever Syndromes" in NEJM

\title{
Sinan Kardeş
}

Corresponding author: Sinan Kardeş, E-mail: sinan.kardes@istanbul.edu.tr,

Department of Medical Ecology and Hydroclimatology, Istanbul Faculty of Medicine, Istanbul University, Istanbul, Turkey

\section{(cc) BY-NC-ND}

Dear Editor,

I read with great interest the valuable article by De Benedetti and colleagues, the leading scientists in the relevant field, about canakinumab for autoinflammatory recurrent fever syndromes, which was recently published in the New England Journal of Medicine (1). I would like to raise some worthwhile issues that need to be clarified.

The "Statistical Analysis Plan" $(2,3)$ of the study stated "all efficacy evaluations will be performed on the full analysis set" and "The Full Analysis Set (FAS) Epoch 2 will consist of all randomized patients in the randomized treatment epoch who received at least one dose of study drug in epoch 2 . Following the intent-to-treat principle, patients will be analyzed according to the treatment they were assigned to at randomization". According to CONSORT 2010 Explanation and Elaboration, "Intention-to-treat analysis corresponds to analysing the groups exactly as randomised", "The simple way to deal with any protocol deviations is to ignore them: all participants can be included in the analysis regardless of adherence to the protocol, and this is the intention-to-treat approach. Thus, exclusion of any participants for such reasons is incompatible with intention-totreat analysis", and "The term 'modified intention-to-treat' is quite widely used to describe an analysis that excludes participants who did not adequately adhere to the protocol, in particular those who did not receive a defined minimum amount of the intervention. An alternative term is "per protocol"' (4). Hence, the study efficacy analysis appears to be modified intention-to-treat analysis, even though the authors incorrectly called an intention-to-treat approach. Interestingly, in the literature, several systematic reviews addressed whether investigators who expressed that an intention-to-treat analysis was used really did what they say $(5,6)$ and found that $13 \%(5)$ $23 \%$ (6) of the reviewed articles did not analyze according to intention-to-treat. This is similarly the case in De Benedetti and colleagues' study (1). The majority of P values in Figure 2 (1) are not matched with the records of ClinicalTrials.gov $(7,8)$. Namely, the primary outcome and all three secondary outcomes in mevalonate kinase deficiency (MKD) and tumor necrosis factor receptor-associated periodic syndrome (TRAPS); and serum amyloid A (SAA) in colchicine-resistant familial Mediterranean fever (crFMF). These discrepancies cannot simply be attributed to rounding numbers.

Last and very least, the number of the actual enrolment in the article (i.e. 181 or 203) (1) is higher than the anticipated sample size (i.e. 180) $(2,3,9)$. According to CONSORT, "If the actual sample size differed from the originally intended sample size for some other reason (for example, because of poor recruitment or revision of the target sample size), the explanation should be given" (4). However, it was surprising to see that the article did not contain any description of sample size and power estimations for the study. More seriously, the authors, in the article, failed to justify why the study recruited more patient than intended.
In conclusion, I believe that the efficacy analysis should be reperformed using the intention-to-treat (all patients as randomized regardless of adherence to the protocol (4) population. Also, I strongly believe that the discrepancies in $\mathrm{P}$ values require immediate clarification.

I end with the Royal Society's motto "Nullius in verba" and with an elegant Viewpoint (10).

Disclosure statement: The author declares that he has no conflict of interest.

Funding: None.

Note: The letter could not be submitted through NEJM online submission system, because it is not possible to submit a letter after three weeks of the publication date of the article. I submitted a presubmission inquiry requesting consideration of the letter for publication. The editors replied that the editorial policy is firm and letters regarding the 17 May issue are no longer being considered.

\section{REFERENCES}

1. De Benedetti F, Gattorno M, Anton J, Ben-Chetrit E, Frenkel J, Hoffman HM, et al. Canakinumab for the Treatment of Autoinflammatory Recurrent Fever Syndromes. N Engl J Med. 2018;378:1908-1919

2. ClinicalTrials.gov number, NCT02059291. Available from: https://clinicaltrials.gov/ProvidedDocs/91/NCT02059291/P rot 000.pdf. Accessed 23 June 2018

3. ClinicalTrials.gov number, NCT02059291. Available from: https:/clinicaltrials.gov/ProvidedDocs/91/NCT02059291/S AP_001.pdf. Accessed 23 June 2018

4. Moher D, Hopewell S, Schulz KF, Montori V, Gøtzsche PC, Devereaux PJ, et al. CONSORT 2010 explanation and elaboration: updated guidelines for reporting parallel group randomised trials. BMJ .2010;340:c869

5. Hollis S, Campbell F. What is meant by intention to treat analysis? Survey of published randomised controlled trials. BMJ. 1999;319:670-4

6. Gravel J, Opatrny L, Shapiro S. The intention-to-treat approach in randomized controlled trials: are authors saying what they do and doing what they say? Clin Trials .2007; $4: 350-6$

7. ClinicalTrials.gov number, NCT02059291. Latest version, April 6, 2018. Available from: https:/clinicaltrials.gov/ct2/history/NCT02059291?V_10= View\#StudyPageTop. Accessed 23 June 2108

8. ClinicalTrials.gov number, NCT02059291. 'Study Results' tab. Available from: https:/clinicaltrials.gov/ct2/show/results/NCT02059291. Accessed 23 June 2108

9. ClinicalTrials.gov number, NCT02059291. November 24, 2014 version. Available from: https:/clinicaltrials.gov/ct2/history/NCT02059291?V 5=Vi ew\#StudyPageTop. Accessed 23 June 2108

10. Lehman RS. Nullius in verba: don't take anyone's word for it. JAMA 